\title{
Successful endovascular treatment of a ruptured bihemispheric posterior inferior cerebellar artery aneurysm: illustrative case
}

\author{
Shingo Nishihiro, MD, Tomotsugu Ichikawa, MD, Yu Takahashi, MD, Yuichi Hirata, MD, Nobuhiko Kawai, MD, Satoshi Kuramoto, MD, \\ Yasuhiro Ono, MD, Yuji Goda, MD, and Masamitsu Kawauchi, MD
}

Department of Neurological Surgery, Kagawa Prefectural Central Hospital, Takamatsu, Kagawa, Japan

BACKGROUND Normal posterior inferior cerebellar artery (PICA) anatomy is highly variable, but bihemispheric PICA crossing the midline to supply the vascular territory of bilateral cerebellar hemisphere is rare. Herein, the authors reported a rare case of ruptured aneurysm that was associated with bihemispheric PICA and successfully treated endovascularly.

OBSERVATIONS A 46-year-old woman presented with sudden headache and loss of consciousness because of an intraventricular hemorrhage due to a ruptured aneurysm that was associated with the bihemispheric PICA. Angiography revealed that the aneurysm was located at the bifurcation between the bihemispheric PICA and the bilateral distal PICA. The ruptured aneurysm was successfully occluded using coil embolization, which preserved the parent artery with no procedural-related complication.

LESSONS To the best of the authors' knowledge, this was the first report of a ruptured aneurysm associated with bihemispheric PICA being successfully treated endovascularly. Aneurysm formation may be accelerated by hemodynamic stress and vascular fragility. For neurosurgeons and neurointerventionalists, it is important to understand the anatomical variation of PICA, especially bihemispheric PICA, which is a potential risk factor for a fatal stroke.

https://thejns.org/doi/abs/10.3171/CASE21367

KEYWORDS bihemispheric PICA; coil embolization; intraventricular hemorrhage; ruptured aneurysm

Aneurysms of the posterior inferior cerebellar artery (PICA) are uncommon, accounting for $0.49 \%$ to $3 \%$ of all intracranial aneurysms. $^{1,2}$ Most PICA-related aneurysms occur at the junction of the vertebral artery (VA) and PICA. Aneurysms arising from the distal PICA are relatively rare, accounting for $15 \%$ to $30 \%$ of all PICA aneurysms. ${ }^{3}$ The normal PICA anatomy is highly variable, and variations include hypoplasia or aplasia, double or duplicated, and extracranial or epidural origin. ${ }^{4,5}$ The unilateral PICA was reported to be absent in $15 \%$ to $25 \%$ cases. $^{6,7}$ When the unilateral PICA shows hypoplasia or aplasia, the PICA vascular territory is almost completely supplied by the ipsilateral anterior inferior cerebellar artery or may be supplied by the contralateral PICA, which is uncommon. This variable anatomy of a single PICA that supplies the vascular territory of both PICAs is rare, and the artery is interpreted as a bihemispheric PICA.
An aneurysm that is associated with the bihemispheric PICA is rare. Herein, we describe a rare case of a ruptured aneurysm that was associated with the bihemispheric PICA and was successfully treated using coil embolization.

\section{Illustrative Case}

A 46-year-old woman presented with sudden headache and loss of consciousness. Initial computed tomography (CT) showed a hematoma in the fourth ventricle (Fig. 1A and B). CT angiography (CTA) detected the saccular aneurysm that was located at the main trunk of the right PICA near the intraventricular hemorrhage (Fig. $1 \mathrm{C}$ and $\mathrm{D}$ ). Digital subtraction angiography (DSA) revealed that the main trunk of the right PICA that crossed the midline supplied bilateral cerebellar hemispheres with absence of the left PICA, and it showed a small

ABBREVIATIONS CT = computed tomography; CTA = CT angiography; DSA = digital subtraction angiography; $\mathrm{MRI}=$ magnetic resonance imaging; $\mathrm{PICA}=$ posterior inferior cerebellar artery; VA = vertebral artery.

INCLUDE WHEN CITING Published August 16, 2021; DOI: 10.3171/CASE21367.

SUBMITTED June 19, 2021. ACCEPTED July 7, 2021.

(C) 2021 The authors, CC BY-NC-ND 4.0 (http://creativecommons.org/licenses/by-nc-nd/4.0/). 

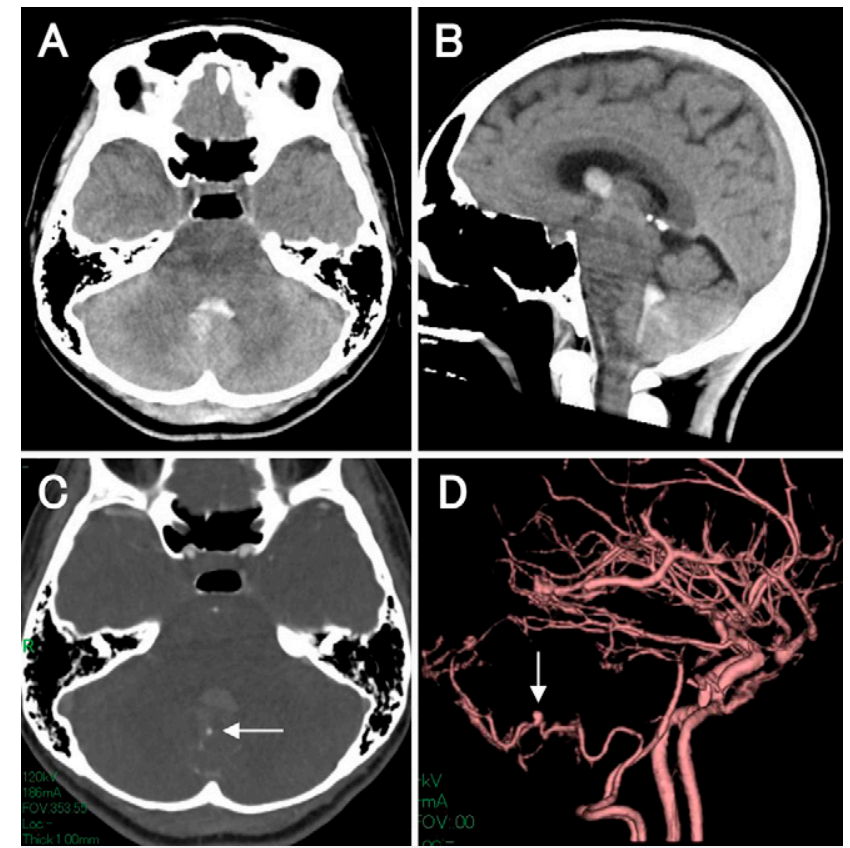

FIG. 1. Initial noncontrasted CT and CTA at admission. Noncontrasted axial (A) and sagittal (B) CT showed a hematoma in the fourth ventricle. CTA axial image (C) and three-dimensional CTA (D) detected a small aneurysm (white arrows) that was located at the main trunk of the right PICA near the fourth intraventricular hemorrhage and was associated with the bleeding. saccular aneurysm $(2.8 \times 2.5 \mathrm{~mm})$ that was located at the bifurcation between the main trunk of the right PICA and bilateral distal PICA (Fig. 2). Endovascular treatment using coil embolization of the ruptured aneurysm was performed under general anesthesia. A 7-Fr Roadmaster guiding catheter (Goodman) was inserted into the right VA. An Excelsior SL-10 microcatheter (Stryker) was inserted into the aneurysm, which was navigated by a CHIKAI 14 microguidewire (Asahi Intecc). The aneurysm was occluded using three coils (one Target 360 nano $2 \mathrm{~mm} \times 3 \mathrm{~cm}$ and two Target 360 nano $1 \mathrm{~mm} \times 2$ $\mathrm{cm}$; Stryker) without any periprocedural complications, and the patency of the main trunk of the right PICA and the bilateral distal PICA were preserved (Fig. 3). Follow-up DSA 7 days after the treatment showed no regrowth of the coiled aneurysm and no vasospasm (Fig. 4). Magnetic resonance image (MRI) 3 weeks after the treatment showed no regrowth or recurrence of the coiled aneurysm and no infarction in the bilateral cerebellar hemisphere (Fig. 5A and B). The woman's clinical course was extremely good, with no vasospasm or hydrocephalus. She was discharged without neurological deficit 4 weeks after treatment. A follow-up MRI 6 weeks after treatment showed that the coiled aneurysm was stable without regrowth or recurrence (Fig. 5C and D), and MRI 6 months after treatment also showed no regrowth or recurrence of the coiled aneurysm (Fig. 5E).

\section{Discussion}

\section{Observations}

Aneurysms arising from the PICA are relatively rare. Aneurysms located on the distal PICA, except for the VA-PICA junction, are especially rare, accounting for $15 \%$ to $30 \%$ of all PICA aneurysms. ${ }^{3}$
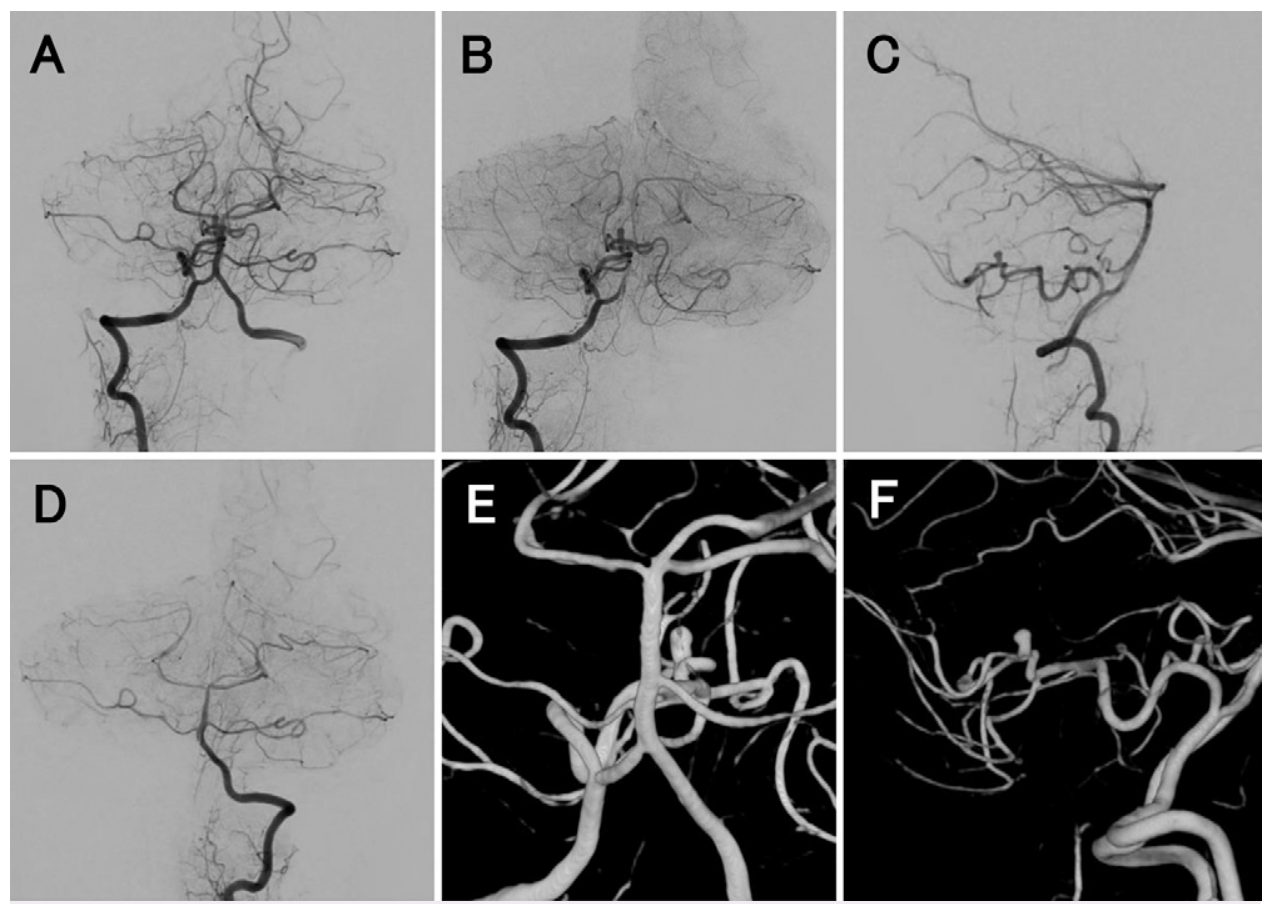

FIG. 2. Preoperative DSA. Anteroposterior (A and B) and lateral (C) right vertebral angiography showed a small saccular aneurysm that was located at the main trunk of the right PICA, and it crossed the midline to supply the vascular territory of the bilateral cerebellar hemispheres D: Left vertebral angiography showed the absence of the left PICA. Anteroposterior (E) and lateral (F) three-dimensional DSA showed a small saccular aneurysm that was located at the bifurcation between the main trunk of the right PICA and the bilateral distal PICA. 

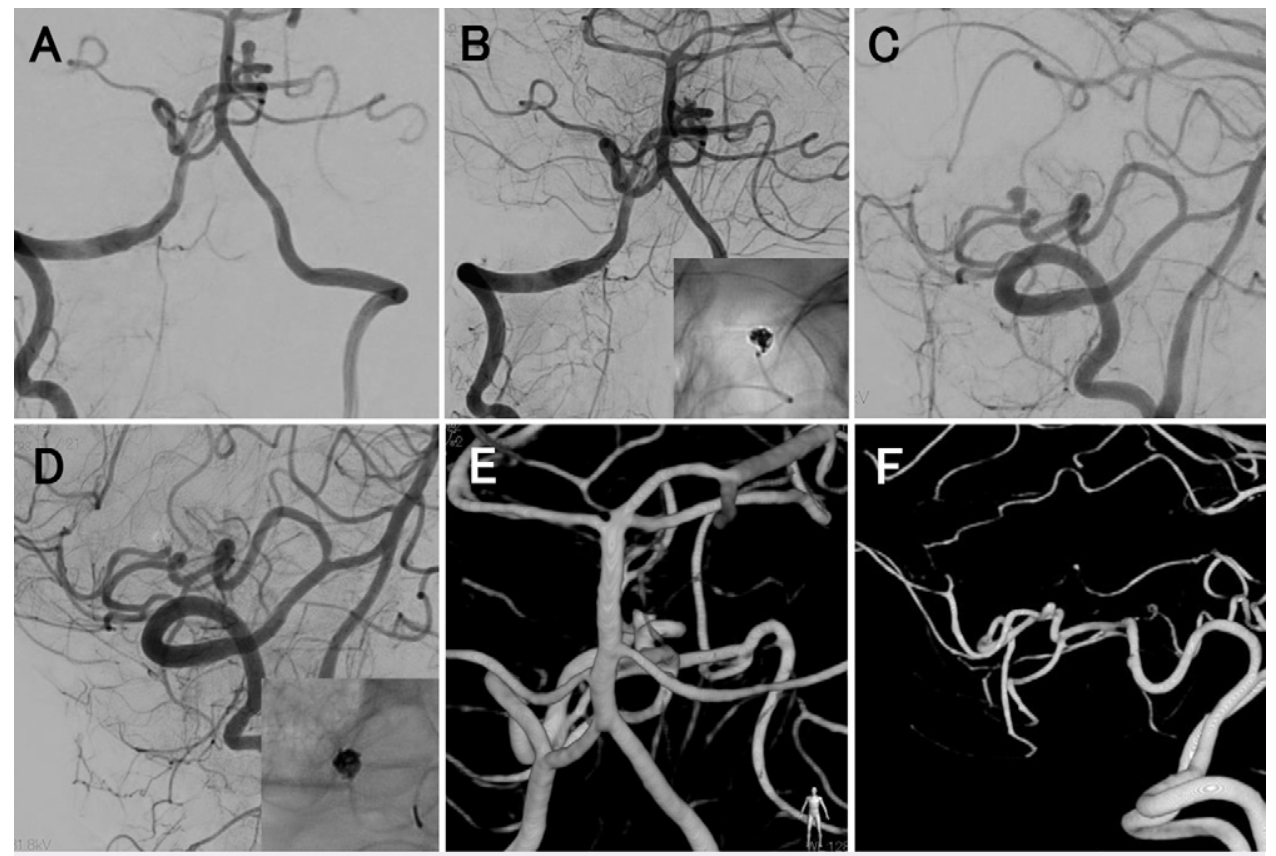

FIG. 3. Coil embolization of the aneurysm. Anteroposterior $(\mathbf{A})$ and lateral $(\mathbf{C})$ views of the right vertebral angiography before treatment. Anteroposterior (B) and lateral (D) views of the right vertebral angiography immediately after treatment showed complete occlusion of the aneurysm with preservation of the bihemispheric PICA and the bilateral distal PICA. Anteroposterior $(\mathbf{E})$ and lateral $(\mathbf{F})$ views of three-dimensional DSA immediately after treatment.

Moreover, it is rare that an aneurysm of the distal PICA is associated with an anatomical variation in the PICA, such as in the present case. In our case, the bihemispheric PICA crossed the midline
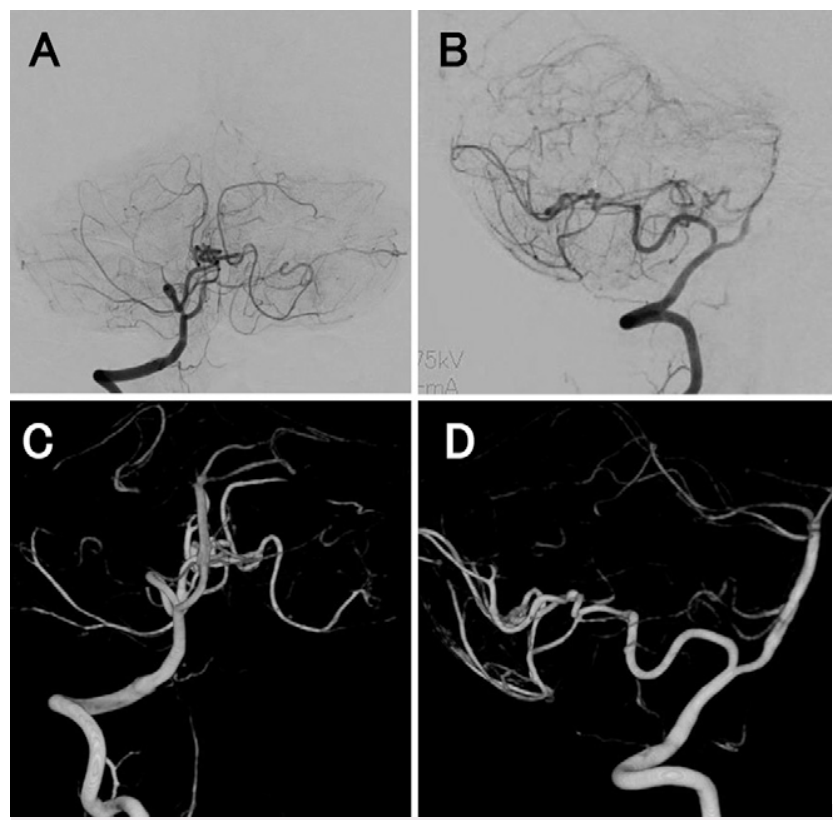

FIG. 4. Follow-up DSA at 7 days after treatment. Anteroposterior (A) and lateral $(\mathbf{B})$ views of right vertebral angiography showed the stability of the coiled aneurysm without regrowth or recurrence. Anteroposterior (C) and lateral (D) views of three-dimensional DSA also showed complete occlusion of the aneurysm without regrowth or recurrence. and supplied both cerebellar hemispheres, and the aneurysm was located at the bifurcation between the bihemispheric PICA and the bilateral distal PICA. To the best of our knowledge, this is the first report of an aneurysm associated with the bihemispheric PICA that was treated successfully using coil embolization.

The incidence of bihemispheric PICA remains unclear. A few reports document a bihemispheric PICA. Cullen et al. reported the incidence of a bihemispheric PICA to be $0.1 \%$ as an incidental finding. ${ }^{8}$ In another report, Carlson et al. reported the incidence to be $3.6 \%$ based on a retrospective angiogram review. ${ }^{9}$

Intradural arteries rarely cross the midline to supply the contralateral area. There are three explanations for why arteries cross the midline. ${ }^{8}$ First, the network of arterial architecture could cross and bridge the midline to supply the neural structure. This resembles the pial network of the dorsal spinal cord. Second, as the artery develops, it crosses a midline structure, and the contralateral territory is thus supplied across the midline, such as in the corpus callosum. A bihemispheric PICA may develop in this manner, using the cerebellar vermis as a midline structure. Finally, the midline artery could supply both sides of the territory after arterial fusion. This pattern is observed in the anterior spinal artery, basilar artery, and limbic arch. 8,10

Several reports indicate that the aneurysms are associated with the bihemispheric PICA. However, those aneurysms were located at the bifurcation of the VA and the bihemispheric PICA. ${ }^{11-14}$ In another report, the dissecting aneurysm was located in the retromedullary segment of the bihemispheric PICA, and it was treated using endovascular parent vessel occlusion followed by suboccipital craniectomy. ${ }^{15}$ It was suspected that parent vessel occlusion of the bihemispheric PICA, which supplied both of the cerebellar hemispheres, 

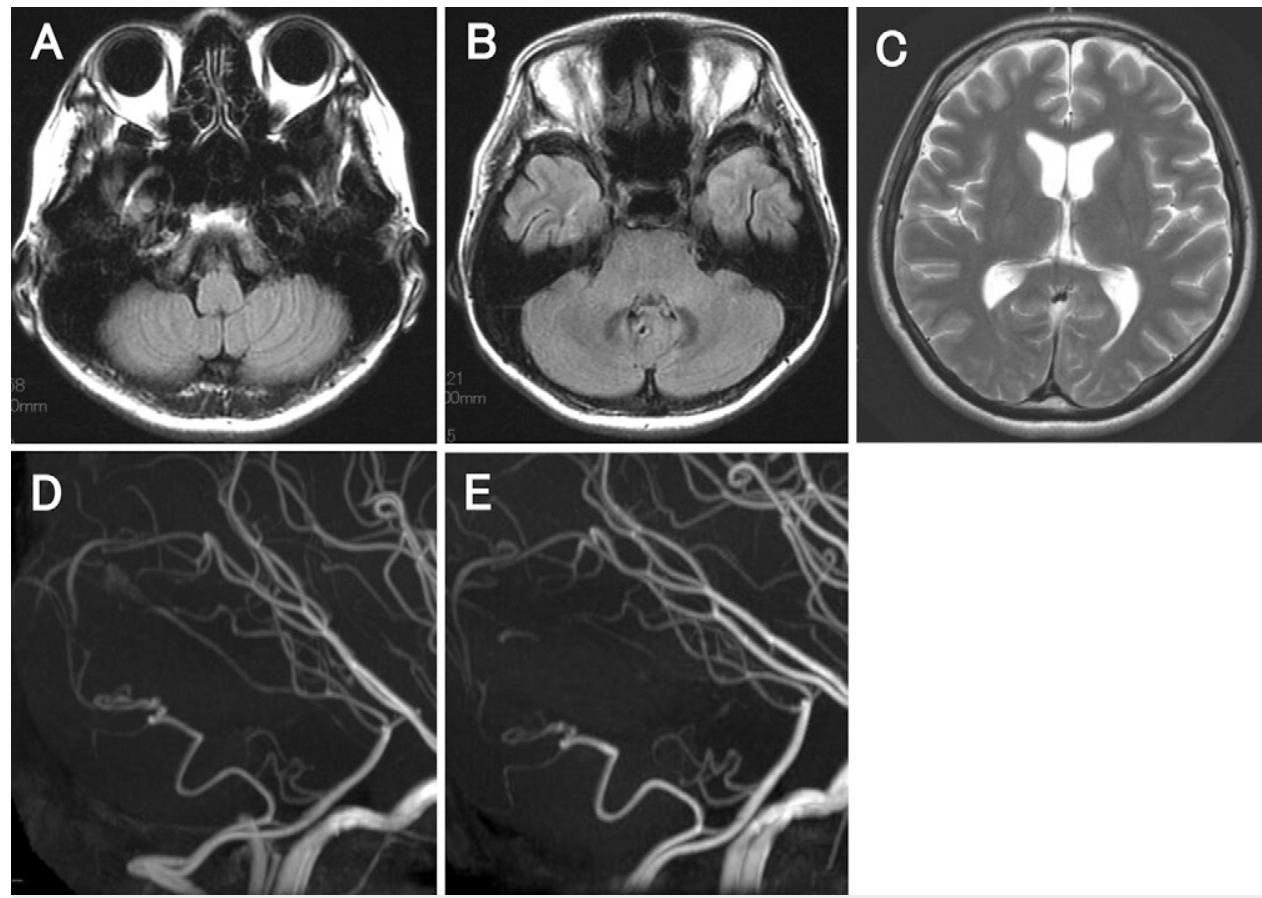

FIG. 5. Follow-up MRI/MR angiography (MRA) after the treatment. A and B: Axial images of fluid-attenuated inversion recovery at 3 weeks after treatment showed no infarction in the bilateral cerebellar hemisphere. C: Axial T2-weighted image at 6 weeks after treatment showed a normal-sized ventricle without hydrocephalus. D: Lateral view MRA at 6 weeks after treatment showed no regrowth or recurrence of the coiled aneurysm.

E: Lateral view MRA at 6 months after treatment revealed no regrowth or recurrence of the coiled aneurysm.

had resulted in extension of the cerebellar infarction. In the present case, coil embolization of the ruptured aneurysm associated with the bihemispheric PICA was performed successfully. The aneurysm that was treated with coil embolization did not regrow or rerupture, leading to aneurysm stability, and the patency of the bihemispheric PICA was preserved.

No proven mechanisms have clearly identified the association between a bihemispheric PICA and aneurysms. Considering that the PICA contralateral vascular territory is supplied by a single PICA due to aplasia of the contralateral VA or PICA, its contribution to the formation of the bihemispheric PICA aneurysm could be explained by the hemodynamic stress. ${ }^{13}$ Moreover, it was reported that $37.5 \%$ of peripheral aneurysms in the posterior fossa were associated with an arteriovenous malformation. ${ }^{16} \mathrm{An}$ arteriovenous malformation associated with a peripheral aneurysm in the posterior fossa developed more frequently than in other locations. Additionally, hemodynamic stress may promote aneurysm formation, especially in the posterior circulation. ${ }^{16}$ In the present case, the aneurysm was located in the bifurcation between the single PICA and the bilateral distal PICA. This finding suggests that hemodynamic forces may contribute to formation of this aneurysm, which is similar to other aneurysms that are located at the bifurcation of the middle cerebral artery.

Another explanation for this aneurysm formation may be the fragility of the vessel wall. ${ }^{13}$ It is well known that distal posterior inferior cerebellar artery aneurysms develop at both vessel bifurcations and turning points. ${ }^{17,18}$ Embryologically, dorsal-side arteries in the medulla oblongata communicate through a primitive lateral basilar-vertebral anastomosis that is present around week 7 of embryonic development. The anastomosis gradually degenerates, and the tortuous point of the vessels persists as the remnant. The tortuous point may be a bifurcation of the vessels at the embryonic stage, which suggests that congenital vascular fragility could be present. ${ }^{19}$ This congenital fragility could also facilitate aneurysm formation because hemodynamic stress may promote aneurysm formation.

In the present case, the aneurysm was successfully occluded by endovascular treatment, and regrowth of the coiled aneurysm was not confirmed in the follow-up period. The pathophysiology of aneurysms arising at this location has not been accurately evaluated. The aneurysm may be considered a dissecting aneurysm because the factors associated with aneurysm formation were hemodynamic stress and vessel wall fragility. ${ }^{13,16,19}$ Considering the aneurysm characteristics, there was the possibility of aneurysm regrowth or recurrence, as had been observed in a previous report. ${ }^{15}$ In such a situation, parent vessel occlusion of the coiled aneurysm, including the affected vessel, may be the best treatment option to prevent a rebleed. Parent vessel occlusion of a distal PICA aneurysm could be a reasonable treatment. Lister et al. divided the PICA trunks into the following five segments based on their relationship to the medulla and the cerebellum: (1) anterior medullary, (2) lateral medullary, (3) tonsillomedullary, (4) telovelotonsillar, and (5) cortical. Few brain perforators arise from the telovelotonsillar segment of the PICA. ${ }^{17,20}$ Therefore, parent vessel occlusion of the anterior and lateral PICA segments should not be performed because there is a risk of medullary infarction due to occlusion of the perforators. Unlike the anterior and lateral PICA segments, however, parent vessel occlusion of the telovelotonsillar 
PICA segment should not have a high risk of medullary infarction because there are fewer perforators to the brainstem.

It is important to understand the anatomical PICA variation when considering the risk of surgery or endovascular treatment. The PICA vascular territory is different with anatomical variations, especially in the present case, in which the bihemispheric PICA supplies both cerebellar hemispheres. In the previous report, the patient had undergone decompressive craniectomy for a bilateral cerebellar infarction after the bihemispheric PICA, which supplied both cerebellar hemispheres, was occluded. $^{15}$ In another report, a bilateral cerebellar infarction occurred because of stenosis of a single PICA that supplied both PICA vascular territories. ${ }^{21}$ Parent vessel occlusion should not be used as a treatment strategy for aneurysms that are located in the main trunk of bihemispheric PICA because bilateral cerebellar infarction due to bihemispheric PICA occlusion is a crucial situation. Considering the possibility of this anatomical PICA variation, it is important to confirm the vascular territory of the contralateral PICA or the absence of a contralateral PICA using preoperative angiography. The risk associated with the treatment for this lesion is high because access to the aneurysm is relatively difficult because of the tortuous vessel or distal site, and preservation of the main bihemispheric PICA trunk is required to prevent bilateral cerebellar infarction. This suggests that preoperative evaluation of angiographic architecture is necessary, especially for this lesion. A detailed understanding of the PICA variation is essential during diagnosis and treatment and can result in the prevention of complications as described above.

\section{Lessons}

A ruptured aneurysm that was associated with a bihemispheric PICA was successfully treated using coil embolization. Aneurysm formation may occur because of hemodynamic stress and vascular fragility. Neurosurgeons and neurointerventionalists should be aware of PICA anatomy, especially bihemispheric PICA, which is a potential risk factor for a fatal stroke.

\section{References}

1. Hudgins RJ, Day AL, Quisling RG, Rhoton AL Jr, Sypert GW, Garcia-Bengochea F. Aneurysms of the posterior inferior cerebellar artery. A clinical and anatomical analysis. $J$ Neurosurg. 1983;58(3):381-387.

2. Locksley HB. Natural history of subarachnoid hemorrhage, intracranial aneurysms and arteriovenous malformations. Based on 6368 cases in the cooperative study. J Neurosurg. 1966;25(2):219-239.

3. Ishikawa T, Suzuki A, Yasui N. Distal posterior inferior cerebellar aneurysms: report of 12 cases. Neurol Med Chir (Tokyo). 1990;30(2):100-108

4. Lasjaunias $P$, Vallee $B$, Person $H$, Ter Brugge K, Chiu M. The lateral spinal artery of the upper cervical spinal cord. Anatomy, normal variations, and angiographic aspects. J Neurosurg. 1985; 63(2):235-241.

5. Lesley WS, Dalsania HJ. Double origin of the posterior inferior cerebellar artery. AJNR Am J Neuroradiol. 2004;25(3):425-427.

6. Margolis MT, Newton TH. The posterior inferior cerebellar artery. In: Newton TH, Potts DG, eds. Radiology of the Skull and Brain. CV Mosby; 1974:1710-1774.

7. Shrontz C, Dujovny M, Ausman Jl, et al. Surgical anatomy of the arteries of the posterior fossa. J Neurosurg. 1986;65(4):540-544.

8. Cullen SP, Ozanne A, Alvarez H, Lasjaunias P. The bihemispheric posterior inferior cerebellar artery. Neuroradiology. 2005; 47(11):809-812.
9. Carlson AP, Alaraj A, Dashti R, Aletich VA. The bihemispheric posterior interior cerebellar artery: anatomic variations and clinical relevance in 11 cases. J Neurointerv Surg. 2013;5(6):601-604.

10. Toms J, Wadhwa R, Ambekar S, Cuellar H. Bihemispheric posterior inferior cerebellar artery occurring with an azygos anterior cerebral artery: case study. Case Rep Radiol. 2014;2014:541081.

11. Sardhara J, Behari S, Patwari S, et al. A low-lying, solitary, bihemispheric PICA with an associated spontaneous vertebral-PICA dissecting aneurysm. Acta Neurochir (Wien). 2013;155(8):1539-1542.

12. Reinacher $P$, Krings $T$, Burgel U, Hans FJ. Posterior inferior cerebellar artery (PICA) aneurysm arising from a bihemispheric PICA. Clin Neuroradiol. 2006;16:190-191.

13. Ogasawara $Y$, Kashimura H, Aso K, Saura H. Subarachnoid hemorrhage due to ruptured intracranial aneurysm arising from a vertebral artery-bihemispheric posterior inferior cerebellar artery bifurcation. J Neurosci Rural Pract. 2017;8(4):654-656.

14. Carlson AP. Tailored PICA revascularization for unusual ruptured fusiform vertebro-PICA origin aneurysms: rationale and case illustrations. J Neurol Surg Rep. 2015;76(2):e275-e278.

15. Tan CL, Anil G, Yeo TT, Chou N. Challenges in the management of a ruptured bihemispheric posterior inferior cerebellar artery aneurysm. World Neurosurg. 2019;122:317-321.

16. Gács G, Viñuela F, Fox AJ, Drake CG. Peripheral aneurysms of the cerebellar arteries. Review of 16 cases. J Neurosurg. 1983;58(1):63-68.

17. Lister JR, Rhoton AL Jr, Matsushima T, Peace DA. Microsurgical anatomy of the posterior inferior cerebellar artery. Neurosurgery. 1982;10(2):170-199.

18. Nishizaki T, Tamaki N, Nishida Y, Fujita K, Matsumoto S. Aneurysms of the distal posterior inferior cerebellar artery: experience with three cases and review of the literature. Neurosurgery. 1985;16(6):829-832.

19. Fujiwara K, Ito J, Kanayama S. Multiple aneurysms of the PICA communicating artery: a case report. Article in Japanese. No Shinkei Geka. 1999;27(2):177-182.

20. Li H, Li XF, He XY, et al. Endovascular treatment of dissecting aneurysms of the posterior inferior cerebellar artery and predictors of outcome. J Stroke Cerebrovasc Dis. 2015;24(9):2134-2142.

21. Gaida-Hommernick B, von Smekal U, Kirsch M, Schminke U, Machetanz J, Kessler C. Bilateral cerebellar infarctions caused by a stenosis of a congenitally unpaired posterior inferior cerebellar artery. J Neuroimaging. 2001;11(4):435-437.

\section{Disclosures}

The authors report no conflict of interest concerning the materials or methods used in this study or the findings specified in this paper.

\section{Author Contributions}

Conception and design: Nishihiro, Takahashi. Acquisition of data: Nishihiro, Ichikawa, Hirata, Kawai, Kuramoto, Ono, Goda, Kawauchi. Analysis and interpretation of data: Ichikawa, Hirata, Kawai, Kuramoto, Ono, Kawauchi. Drafting the article: Nishihiro, Ichikawa, Hirata, Kawai, Kuramoto, Ono, Kawauchi. Critically revising the article: Nishihiro, Ichikawa. Reviewed submitted version of manuscript: Nishihiro, Ichikawa, Goda. Administrative/technical/material support: Nishihiro, Takahashi. Study supervision: Ichikawa.

\section{Correspondence}

Shingo Nishihiro: Kagawa Prefectural Central Hospital, Takamatsu, Kagawa, Japan. n.shingo777@gmail.com. 